\title{
Z-bump Pulsations in Helium Stars
}

\author{
C. Simon Jeffery \\ Armagh Observatory, College Hill, Armagh BT61 9DG, Northern \\ Ireland
}

Hideyuki Saio

Astronomical Institute, School of Sciences, University of Tohoku, Sendai 980, Japan

\begin{abstract}
Radial and nonradial pulsations are excited in low-mass helium stars with effective temperatures between 7000 and $35000 \mathrm{~K}$. In the case of the most luminous stars, these are driven by strange-mode instabilities (Saio \& Jeffery 1988). Less luminous helium stars are mostly non-variable, except around $25000 \mathrm{~K}$. V652 Her is the prototype radially pulsating helium star, with $T_{\text {eff }} \sim 25000 \mathrm{~K}$ and a pulsation period of 0.108 (Hill et al. 1981). The pulsation was only understood to be driven by the $\kappa$-mechanism with the introduction of Z-bump opacity (Saio 1993).

The importance of Z-bump instability in low-mass helium stars has been investigated further through a series of pulsation models (Jeffery \& Saio 1999a). It is shown that Z-bump pulsations can persist to surprisingly high hydrogen abundances in low-mass stars, and may consequently be excited in several helium-rich hot subdwarfs with $T_{\text {eff }} \sim 25000 \mathrm{~K}$. Within the Z-bump instability region, both radial and nonradial pulsations are excited, and multi-periodic variability is expected.

Pulsation properties are closely linked to the global dimensions of a pulsating star by the period - mean density relation. When spectroscopic measurements are also available, the twin constraints of period and surface gravity can place very tight limits on the stellar radius (Jeffery \& Saio 1999b). These are in excellent agreement with independent measurements for V652 Her and place a lower limit on the mass of the recently discovered Z-bump pulsator BX Cir (Kilkenny et al. 1999).
\end{abstract}

\section{References}

Hill, P. W., Kilkenny, D., Schönberner, D., \& Walker, H. 1981, MNRAS, 197, 81

Jeffery, C. S. \& Saio, H. 1999a, MNRAS, 308, 221

Jeffery, C. S. \& Saio, H. 1999b, MNRAS (submitted)

Kilkenny, D., Koen, C., Jeffery, C. S., Hill, N. C., \& O'Donoghue, D. 1999, MNRAS, in press

Saio, H. 1993, MNRAS, 260, 465

Saio, H. \& Jeffery, C. S. 1988, ApJ, 328, 714 